\title{
Development of IgY-Based Sandwich ELISA as a Robust Tool for Rapid Detection and Discrimination of Toxigenic Vibrio cholerae
}

\author{
Mahdiye Bayat, ${ }^{1,2}$ Alireza Khabiri $\mathbb{D},{ }^{2}$ and Behzad Hemati ${ }^{1}$ \\ ${ }^{1}$ Department of Microbiology, Islamic Azad University-Karaj Branch, Karaj 3148635731, Iran \\ ${ }^{2}$ Diagnostic Biotechnology Unit, Pasteur Institute of Iran, Research and Production Complex, Karaj, Iran \\ Correspondence should be addressed to Alireza Khabiri; khabiri@pasteur.ac.ir
}

Received 26 March 2018; Revised 10 August 2018; Accepted 19 August 2018; Published 2 October 2018

Academic Editor: José A. Oteo

Copyright ( $\odot 2018$ Mahdiye Bayat et al. This is an open access article distributed under the Creative Commons Attribution License, which permits unrestricted use, distribution, and reproduction in any medium, provided the original work is properly cited.

Background. The conventional methods for diagnosis of Vibrio cholerae are time consuming, complicated, and expensive. Development of rapid detection tests is critical for prevention and management of cholera. This study aimed to introduce two sensitive sandwich ELISAs based on avian antibodies (IgY) targeting outer membrane protein W (OmpW) and cytotoxin B (CtxB) antigens of $V$. cholerae. Methods. The sequences of ompW and $c t x B$ genes were cloned into pET28a vector. Escherichia coli BL21 (DE3) was transformed with the recombinant vectors, and gene expression was induced by IPTG. The expressed proteins were purified by affinity chromatography using Ni-NTA resins. Two groups of white Leghorn chickens were immunized by recombinant proteins, and the generated antibodies were purified from egg yolks of chickens by PEG precipitation. The antibodies were used for the development of $\alpha$-OmpW and $\alpha$-CtxB ELISAs. Results. The expression and purification yielded 59 and $38 \mathrm{mg}$ of recombinant $\mathrm{OmpW}$ and $\mathrm{CtxB}$, respectively, per one liter of bacterial culture. PEG precipitation and purification of egg yolk antibodies yielded on average $( \pm \mathrm{SD}) 66.5 \pm 1.80$ and $50.9 \pm 2.23 \mathrm{mg}$ of purified $\alpha$-OmpW and $\alpha$-CtxB per egg, respectively. The analytical sensitivity of $\alpha$-OmpW ELISA was $103 \mathrm{cfu} / \mathrm{mL}$ of $V$. cholerae and that of $\alpha$-CtxB ELISA was $33 \mathrm{pg} / \mathrm{mL}$ of recombinant cytotoxin B. The two developed ELISAs did not show any cross-reactivity to any tested bacteria grown in common conditions. Discussion. The current study is the first report on using IgY for detection of $V$. cholerae. The developed ELISAs were shown to have considerable analytical sensitivity and specificity. Therefore, the assays can be one of the convenient methods for sensitive and specific detection of toxigenic $V$. cholerae strains in clinical and environmental samples.

\section{Introduction}

Cholera is an important life-threatening diarrheal disease, transmitted through contaminated water and food. The causative agent of this disease is Vibrio cholerae, which emerged from Southwest Asia. There are around 200 different serogroups of $V$. cholerae, among which $\mathrm{O} 1$ and $\mathrm{O} 139$ are toxigenic [1]. Based on A, B, and C antigens in lipopolysaccharide, the $\mathrm{O} 1$ is classified into three different serotypes, Inaba, Ogawa, and Hikojima. In addition, based on some biological and biochemical properties, $\mathrm{O} 1$ is classified into two biotypes of Classic and El Tor [2].

The conventional diagnostic methods for detection of $V$. cholerae include the isolation and characterization of the causative agent. These laboratory tests are time consuming, labor-intensive, and expensive. On the other hand, lack of required facilities in small laboratories in remote areas makes the detection of organism difficult. In addition, there are some molecular methods for the detection of toxigenic $V$. cholerae, with high sensitivity and specificity $[3,4]$. However, these methods have two fundamental problems: (1) they are expensive and require laboratory facilities and trained personnel and (2) presence of a gene coding exotoxin does not necessarily prove the production and secretion of the relevant protein. Hence, as the severity and rapid spread of the disease make it a serious potential problem, rapid, precise, and on time detection of toxigenic strains in clinical and environmental samples provide an important tool to monitor, control, and prevent the spread of the disease. Therefore, efforts have been made to develop rapid and 
simple diagnostic methods with acceptable sensitivity and specificity [5]. Among the rapid diagnostic methods, coagglutination, ELISA, and dipstick ELISAs are the most important ones used in detection of $V$. cholerae in clinical and environmental samples [6-9].

As the sequence of outer membrane protein $\mathrm{W}(\mathrm{omp} W)$ gene is conserved among different serogroups of $V$. cholerae, species-specific identification of the organism is possible by targeting OmpW antigens in immunoassays [10]. In addition, the strains harboring toxin genes $(\operatorname{ct} x A B)$ are the only producers of the toxin and are known as toxigenic [2]. Therefore, the presence of OmpW and CtxB antigens indicates toxigenic strains of $V$. cholerae.

In the present study, the IgY technology has been employed for the production of specific antibodies against $\mathrm{OmpW}$ and $\mathrm{CtxB}$ antigens of $V$. cholerae. The produced antibodies were used for development of two sandwich enzyme-linked immunosorbent assays $(\alpha$-OmpW ELISA and $\alpha$-CtxB ELISA) for the sensitive and specific detection of toxigenic Vibrio cholerae strains, for direct use in clinical or environmental samples.

\section{Materials and Methods}

2.1. Bacterial Strains. The bacterial strains used in this study were obtained from the microbial collection of Pasteur Institute of Iran and Culture Collection for Research and Industrial Microorganisms of Iranian Research Organization for Science and Technology (IROST).

The bacterial strains used for the evaluation of the specificity of the developed assays are listed in Table 1. Some of these bacteria are part of gastrointestinal (GI) microbiota and some are pathogenic agents of GI. As probability of the presence of the selected organisms in human fecal is high, they can be considered as potential source of cross-reactivity; therefore, they are used for evaluation of the specificity of the developed assays.

2.2. Polymerase Chain Reaction for Amplification of ompW and ctxB Genes. Genomic DNA from Vibrio cholerae O1 strain (Inaba) (PTCC No.: 1611) was extracted using GF-1 Bacterial DNA Extraction Kit (Vivantis, Malaysia, HQ). Based on published sequences (ompW sequence GenBank accession no. X51948 and cholera toxin B encoding $\operatorname{ct} x B$ gene sequence GenBank accession no. X00171), primer sets for the amplification of omp $W$ and $c t x B$ genes were designed (Table 2). PCR amplification of the target DNA was carried out with a reaction volume of $50 \mu \mathrm{l}$. Each reaction mixture contained $2 \mu \mathrm{l}$ of template DNA $(10 \mu \mathrm{g} / \mathrm{mL}), 2 \mu \mathrm{l}$ of each primer $(10 \mu \mathrm{M})$, and $44 \mu \mathrm{l}$ of nuclease-free water in AccuPower ${ }^{\circledR}$ Pfu PCR PreMix tubes (Bioneer, Korea). The reaction mixture was subjected to 30 cycles of denaturation at $94^{\circ} \mathrm{C}$ for $30 \mathrm{~s}$, annealing at $60^{\circ} \mathrm{C}$ and $55^{\circ} \mathrm{C}$ (for omp $W$ and $\operatorname{ct} x B$, respectively) for $30 \mathrm{~s}$, and extension at $72^{\circ} \mathrm{C}$ for 45 and $30 \mathrm{~s}$ (for $\operatorname{omp} W$ and $c t x B$, respectively). Before initiation of the first cycle, the reaction mixture was heated at $94^{\circ} \mathrm{C}$ for 5 min to allow complete denaturation of the template and after the last cycle, the reaction mixture was heated at $72^{\circ} \mathrm{C}$
TABLE 1: Bacterial strains used to evaluate the specificity of $\alpha$-OmpW ELISA.

\begin{tabular}{lc}
\hline Strain & Number \\
\hline Acinetobacter baumannii & PTCC: 1797 \\
Aeromonas hydrophila & MCI: 1096 \\
Bacillus cereus & PTCC: 1154 \\
Bacteroides fragilis & MCI:1011 \\
Enterobacter aerogenes & PTCC: 1221 \\
Enterococcus faecalis & PTCC: 1237 \\
Escherichia coli & PTCC: 1395 \\
Escherichia coli EPEC(M)O55:K 59 & PTCC: 1269 \\
Klebsiella oxytoca & PTCC: 1402 \\
Lactobacillus acidophilus & PTCC: 1643 \\
Proteus mirabilis & PTCC: 1776 \\
Pseudomonas aeroginosa & PTCC: 1310 \\
Salmonella enterica & PTCC: 1709 \\
Shigella dysenteriae & PTCC: 1188 \\
Yersinia enterocolitica & PTCC: 1151 \\
Escherichia coli (ETEC) & PTCC: 1399 \\
\hline
\end{tabular}

TABLE 2: The primer sets used for amplification of $\operatorname{omp} W$ and $\operatorname{ct} x B$ genes.

\begin{tabular}{ll}
\hline \multirow{2}{*}{$\operatorname{sm} W$} & $\begin{array}{l}\text { Forward: aaggatccATGAAACAAACCATTTGC } \\
\text { Reverse: tctctcgagTTAGAACTTATAACCACCC }\end{array}$ \\
\hline \multirow{2}{*}{$x \mathrm{~B}$} & $\begin{array}{l}\text { Forward: aaggatccATTAAATTAAAATTTGGTG } \\
\text { Reverse: atctcgagTTAATTTGCCATACTAATTG }\end{array}$ \\
\hline
\end{tabular}

Underlined nucleotides are the restriction sites of BamHI and XhoI.

for $10 \mathrm{~min}$ to allow complete extension of the PCR products. The PCR products were evaluated on 1\% agarose gel.

2.3. Cloning, Expression, and Purification of Recombinant OmpW and CtxB Proteins. The purified PCR product of ompW and $c t x B$ genes were digested by BamHI and XhoI restriction enzymes and directionally cloned into BamHIand XhoI-digested pET-28a, and the constructs were transformed to $\mathrm{CaCl}_{2}$-treated $\mathrm{BL} 21$ (DE3) strain of Escherichia coli. Transformants were selected and confirmed by the restriction digestion of their plasmids, as well as by colony PCR by the specific primers of each gene. The positive clones were induced to express recombinant proteins by a $4 \mathrm{~h}$ induction with $0.5 \mathrm{mM}$ IPTG. The induced proteins were evaluated by SDS-PAGE analysis. The His-tagged recombinant proteins were purified by Ni-NTA chromatography according to the manufacturer's protocol (Qiagen, Germany). The concentration of purified proteins was determined by the method of Bradford [11].

2.4. Immunization of Chickens. Six 25-week-old white Leghorn chickens were obtained from the laboratory animal production center of the Pasteur Institute of Iran (Alborz, Karaj, Iran). The institutional and national guide for the care and use of laboratory animals was followed. The chickens were divided into three different groups. Immunization of the first and the second group was performed by 
intramuscular injection of $500 \mu \mathrm{l}$ of $10 \mu \mathrm{g} / \mathrm{mL}$ of recombinant $\mathrm{OmpW}$ and $\mathrm{CtxB}$, respectively, formulated with equal volume of complete Freund's adjuvant administrated into two sides of the chest area. Two subsequent boosters containing each recombinant protein emulsified with an equal volume of incomplete Freund's adjuvant and pure recombinant proteins were given on days 30 and 60, respectively. The last group (control group) was administered by PBS ( $\mathrm{pH}$ 7.2) intramuscularly. One week after the last immunization, the eggs were collected daily, for two months, and stored at $4^{\circ} \mathrm{C}$.

\subsection{Purification of $\alpha-O m p W$ and $\alpha-C t x B$ Antibodies from $\mathrm{Egg}$} Yolks of Immunized Chickens. IgY antibodies were extracted from egg yolks using Pauly et al. method [12]. Briefly, twice the volume of yolk suspensions were mixed with PBS and then 3.5\% (w/v) PEG 6000 (Merck, Germany) were added, and the suspension was shaken for $10 \mathrm{~min}$ at room temperature. Two different phases were separated by $20 \mathrm{~min}$ centrifugation at $13,000 \times \mathrm{g}$ at $4^{\circ} \mathrm{C}$. To remove the lipids, the collected supernatant was filtered by Whatman filter paper and $8 \%$ PEG 6000 was added to the filtrate. After shaking and centrifugation as described above, the pellet containing IgY was dissolved in $1 \mathrm{~mL}$ of PBS and the final volume was made to $10 \mathrm{~mL}$ by PBS. Then, $12 \%$ PEG 6000 was added to the solution, shaken by rotation, and centrifuged as described above. The pellet was resuspended carefully in $800 \mu \mathrm{L}$ PBS. The final extract was dialyzed overnight against normal saline with 3 exchanges. The concentration of the purified antibodies was evaluated by Bradford assay [11].

\subsection{Evaluating the Reactivity of the Purified Antibodies.} The specific activity of purified antibodies $(\alpha-\mathrm{OmpW}$ and $\alpha$-CtxB) was evaluated by Western blotting and indirect ELISA.

2.6.1. Western Blotting. The purified OmpW and $\mathrm{CtxB}$ proteins were run on $12 \%$ SDS-PAGE gel and then electrotransferred to nitrocellulose membranes. The membranes were blocked with $1 \%$ bovine serum albumin (BSA) in PBS, overnight at $4^{\circ} \mathrm{C}$. After washing twice with PBS buffer containing $0.1 \%$ Tween 20 (PBS-T), each membrane was incubated with the corresponding purified antibody $(\alpha-\mathrm{OmpW}$ or $\alpha$-CtxB) at a dilution of $1: 3,000$ for 1 hour at room temperature. After extensive washing, the membranes were incubated with goat anti-chicken IgY-HRP (AbcamUK) at a dilution of $1: 4,000$ for 1 hour and, after washing, the blots were developed by $\mathrm{DAB}$ and $\mathrm{H}_{2} \mathrm{O}_{2}$.

2.6.2. Indirect Enzyme-Linked Immunosorbent Assay. The antibodies, purified from the collected eggs at the end of each week after immunization, were tested by indirect ELISA (16 eggs for each immunization group: $\alpha$-OmpW, $\alpha$-CtxB, and control). For this aim, a solution of purified antibodies $(1 \mathrm{mg} / \mathrm{ml})$ were diluted to $1: 10,000$ and $100 \mu \mathrm{l}$ of the diluted antibodies was incubated for 1 hour at $37^{\circ} \mathrm{C}$, in microtiter plates coated with $2 \mu \mathrm{g} / \mathrm{ml}$ of recombinant OmpW or CtxB.
After washing thrice with PBS-T, the wells were incubated with $100 \mu \mathrm{l}$ of IgY goat anti-chicken IgY-HRP (Abcam-UK) at a dilution of $1: 30,000$ for 1 hour at $37^{\circ} \mathrm{C}$. Subsequently, the plates were washed and the colorimetric reaction was carried out using $100 \mu \mathrm{l}$ of tetramethylbenzidine (TMB). The enzymatic reaction was stopped by adding $50 \mu \mathrm{l}$ of $1 \mathrm{M} \mathrm{HCl}$, and the absorbance of each well was measured at $450 \mathrm{~nm}$.

2.7. $\alpha$-OmpW ELISA for Detection of V. cholerae. In order to optimize $\alpha$-OmpW ELISA, bacterial suspensions of $V$. cholerae (PTCC No.: 1611) and E. coli (PTCC No.: 1395) with the density equivalent to $1 \mathrm{McF}$ arland standard were used as positive and negative controls, respectively. A part of the dialyzed $\alpha$-OmpW antibodies was diluted to $10,5,2.5,1.25$, $0.6,0.3$, and $0.15 \mu \mathrm{g} / \mathrm{mL}$ in coating buffer $(100 \mathrm{mM}$ sodium carbonate-bicarbonate buffer with $\mathrm{pH}$ 9.6). Another part of dialyzed $\alpha-\mathrm{OmpW}$ antibodies was conjugated with horseradish peroxidase (Sigma Aldrich, USA) by sodium periodate method [13] to use as detection antibody. HRPconjugated OmpW IgY was diluted to $1: 1000,1: 2000,1$ : $4000,1: 8000,1: 16,000,1: 32,000$ and $1: 64,000$ in $1 \%$ BSA in PBS buffer. Checkerboard titration was performed to determine the optimal amounts of detection and capture $\alpha$-OmpW antibodies. The positive and negative controls were tested in triplicate, and the mean OD of them was used for evaluation.

For $\alpha$-OmpW ELISA, the microplates (Nunc, Denmark) were coated with $100 \mu \mathrm{l}$ of purified $\alpha$-OmpW antibodies $(1.25 \mu \mathrm{g} / \mathrm{mL})$ at $4^{\circ} \mathrm{C}$, overnight. After washing twice with PBS-T, the microplates were blocked for 1 hour at room temperature with PBS containing 1\% BSA. Then, 100 microliter of each bacterial suspension was added to each well and incubated at $37^{\circ} \mathrm{C}$ for 1 hour. The microplates were washed four times with PBS-T, and $100 \mu \mathrm{l}$ of $1: 16,000$ diluted HRP-conjugated $\alpha$-OmpW antibodies was added to wells. After 1-hour incubation at $37^{\circ} \mathrm{C}$ and four times of washing, $100 \mu \mathrm{l}$ of TMB was added to each well and the plates were incubated at room temperature, avoiding direct light. The enzymatic reaction was stopped after 15 minutes by adding $50 \mu \mathrm{l}$ of $1 \mathrm{M} \mathrm{HCl}$. The optical density of wells was read at $450 \mathrm{~nm}$. The mean OD of two blank wells was subtracted from the OD value of sample wells.

2.8. $\alpha$-CtxB ELISA for Detection of Cholera Toxin. In order to optimize $\alpha$-CtxB ELISA, the bacterial supernatants of $V$. cholera (PTCC No.: 1611) and E. coli (PTCC No.: 1395) were cultured under AKI-SW condition to stimulate cytotoxin production [14], and the supernatant of $V$. cholerae and $E$. coli culture media were used as positive and negative controls, respectively. For the development of $\alpha$-CtxB ELISA, a part of dialyzed $\alpha$-CtxB antibodies was diluted to 10, 5, 2.5, $1.25,0.6,0.3$, and $0.15 \mu \mathrm{g} / \mathrm{mL}$ in coating buffer. Then, the biotin-conjugated monoclonal antibody against $V$. cholerae toxin B subunit (Novus Biologicals, USA) was diluted to 1 : $1000,1: 2000,1: 4000,1: 8000,1: 16,000,1: 32,000$ and 1 : 64,000 in $1 \%$ BSA in PBS buffer. Checkerboard titration was performed to determine the optimal amounts of detection and capture $\alpha$-CtxB antibodies. The positive and negative 
controls were tested in triplicate, and the mean OD of them was used for evaluation.

For $\alpha$-CtxB ELISA, the microplates (Nunc, Denmark) were coated with $100 \mu \mathrm{l}$ of purified $\alpha$-CtxB antibodies $(0.6 \mu \mathrm{g} / \mathrm{mL})$ at $4^{\circ} \mathrm{C}$, overnight. After washing twice with PBS-T, the microplates were blocked for 1 hour at room temperature with PBS containing 1\% BSA. Then, 100 microliters of each sample was added to corresponding wells and incubated at $37^{\circ} \mathrm{C}$ for 1 hour. The microplates were washed four times with PBS-T, and $100 \mu \mathrm{l}$ of $1: 32,000$ biotin-conjugated anti- $V$. cholerae toxin $\mathrm{B}$ subunit monoclonal antibody (Novus Biologicals, USA) was added to wells and incubated at $37^{\circ} \mathrm{C}$ for 1 hour. After 1 -hour incubation at $37^{\circ} \mathrm{C}$ and 4 times washing, $1: 5000$ dilution of HRP-conjugated streptavidin (Sigma Aldrich, USA) was added to each well and incubated for 1 hour at $37^{\circ} \mathrm{C}$. Then, the plates were washed for 4 times, $100 \mu \mathrm{l}$ of TMB was added to each well, and the plates were incubated at room temperature avoiding direct light. The enzymatic reaction was stopped after 15 minutes by adding $50 \mu \mathrm{l}$ of $1 \mathrm{M} \mathrm{HCl}$. The optical density of wells was read at $450 \mathrm{~nm}$. The mean OD of the two blank wells was subtracted from the OD value of sample wells.

2.9. Determination of the Analytical Sensitivity of the Developed Assays. The sensitivity of the developed ELISAs was determined by testing tenfold serial dilutions of 0.5 McFarland equivalence suspension of $V$. cholerae $\mathrm{O} 1$ culture ranging from $1.5 \times 10^{8}$ to $1.5 \times 10^{1}$ colony-forming units/mL (for $\alpha$-OmpW ELISA) and twofold serial dilutions of recombinant $\mathrm{CtxB}$ ranging from $1000 \mathrm{pg} / \mathrm{mL}$ to $7.8 \mathrm{pg} / \mathrm{mL}$ (for $\alpha$-CtxB ELISA). Then, the $\alpha$-OmpW and $\alpha$-CtxB ELISAs were run as described in Sections 2.7 and 2.8 , respectively. In each plate, 20 wells were considered as blank and $100 \mu \mathrm{l}$ of dilution buffer (1\% BSA in PBS) was added to blank wells instead of antigen. The optical density of each well was read at $450 \mathrm{~nm}$. The mean and standard deviation of 20 blank wells were determined. Then, the LoB (Limit of Blank) and the absorbance of LoD (Limit of Detection) were calculated for each assay using the following formulas $[15,16]$ :

$$
\begin{aligned}
\mathrm{LOB} & =\operatorname{Mean}_{\text {Blank }}+1.645\left(\mathrm{SD}_{\text {Blank }}\right), \\
\mathrm{Ab}_{\mathrm{LOD}} & =\operatorname{Mean}_{\text {Blank }}+3\left(\mathrm{SD}_{\text {Blank }}\right) .
\end{aligned}
$$

The standard curve and fitted equation of serial dilutions were used to determine the limit of detection of two assays.

2.10. Determination of the Analytical Specificity of the Developed Assays. The specificity of the two assays was determined by testing the supernatant of different bacterial strain (Table 1) cultures with concentration equivalent to 1 McFarland by $\alpha$-OmpW and $\alpha$-CtxB ELISAs, as described above.

E. coli (ETEC) (PTCC: 1399) was used for further evaluation of possible cross-reactivity of heat-labile (LT) toxin of ETEC and $\alpha$-CtxB ELISA. The method suggested by Letícia B. Rocha et al. using lincomycin and triton X-100 was performed for production and release of LT toxin [17], and the supernatant of the culture media was tested by $\alpha$-CtxB ELISA.

2.11. Evaluation of the Analytical Sensitivity and Specificity of the Assays in Spiked Stool Matrix Sample. Five different formed human stool samples were used as matrices for spiking with $V$. cholerae (PTCC No.: 1611) and recombinant $\mathrm{CtxB}$. These samples had been tested negative for enteropathogenic bacteria (Shigella spp., Salmonella spp., Aeromonas sp., Plesiomonas sp., and Yersinia enterocolitica) by routine culture. After that, the samples were pooled to use as stool matrix for further processing.

A bacterial suspension of $V$. cholerae (PTCC No.: 1611) with the density equivalent to $0.5 \mathrm{McF}$ arland was prepared in PBS, then tenfold serial dilutions, ranging from $1.5 \times 10^{8}$ to $1.5 \times 10^{2} \mathrm{cfu} / \mathrm{mL}$, were prepared from this suspension. A stock solution of recombinant $\mathrm{CtxB}$ with the final concentration of $1000 \mathrm{pg} / \mathrm{ml}$ was prepared in PBS, and twofold serial dilutions, ranging up to $15.6 \mathrm{pg} / \mathrm{ml}$, were prepared from the stock solution.

Five grams of the pooled stool matrix was diluted with PBS to a final volume of $30 \mathrm{~mL} .500 \mu \mathrm{L}$ of the diluted stool matrix was then combined with the same volume of the prepared $\mathrm{CtxB}$ and bacterial suspensions to create the following rice-water-stool-like consistency samples:

(i) Seven serial dilutions containing $7.5 \times 10^{7}$ to $7.5 \times 10^{1} \mathrm{cfu} / \mathrm{mL}$ of $V$. cholerae

(ii) Seven serial dilutions containing 500 to $7.8 \mathrm{pg} / \mathrm{mL}$ of recombinant $\mathrm{CtxB}$

All the prepared spiked stool specimens and a sample without spiking, as negative control, were extracted by the following procedure; $500 \mu \mathrm{L}$ of each sample was mixed thoroughly with $500 \mu \mathrm{L}$ of extraction buffer, including $2 \%$ BSA in PBS and $0.02 \%$ sodium azide as a preservative. After vortexing, the samples were centrifuged at $3000 \times g$ for 5 minutes, and the supernatants were transferred into fresh microtubes. The cleared samples were analyzed in duplicate by $\alpha$-OmpW and $\alpha$-CtxB ELISAs, as described in Sections 2.7 and 2.8, respectively. The average optical density of each sample was used for the evaluation of the sensitivity and specificity of the two ELISA methods in spiked stool matrix samples. For this purpose, the samples with an optical density (OD) greater than the limit of detection of two assays, calculated in Section 2.9, were defined as positive.

2.12. Statistical Analysis. All the statistical analyses were performed using SPSS 16.0 statistical software. Significant differences between means of ELISA signals for the determination of specific IgY antibodies were detected by $t$ test. The means of ELISA signals between weeks after immunization were compared by repeated measure ANOVA. $P$ value $<0.05$ was considered as statistically significant.

\section{Results}

3.1. Amplification and Cloning of ompW and ctxB Genes. 
Electrophoresed and ethidium bromide-stained PCR products of both genes showed the expected sizes of 651 and $372 \mathrm{bp}$ for omp $W$ and $c t x B$ genes, respectively. Transformation of ligation products into $E$. coli leads to the growth of kanamycin-resistant colonies, indicating the presence of recombinant vectors in BL21 (DE3) bacteria. Colony PCR and enzymatic digestion of recombinant vectors confirmed correct ligation of inserts into vectors.

3.2. Expression and Purification of Recombinant OmpW and CtxB Proteins. SDS-PAGE analysis of IPTG-induced bacteria revealed the expected $27 \mathrm{kDa}$ band for OmpW and $17 \mathrm{kDa}$ band for $\mathrm{CtxB}$ protein. The final yield of purified recombinant $\mathrm{OmpW}$ and $\mathrm{CtxB}$ was 59 and $38 \mathrm{mg}$ per one liter of bacterial culture, respectively.

3.3. Immunization and $\operatorname{Ig} Y$ Purification. After immunization, no decline in egg-laying capacity was seen $(P$ value $<0.05)$ and the immunized chickens still laid an average of one egg per day, showing no adverse effects or laying decrease caused from immunization. Extraction and purification of specific antibodies were successfully carried out by PEG 6000 . The average yield $( \pm S D)$ of purified antibodies was $66.5 \pm 1.80 \mathrm{mg} \alpha$-OmpW and $50.9 \pm 2.23 \mathrm{mg} \alpha$-CtxB per egg.

3.4. Evaluation of Purified IgY Antibodies. Binding capability of the purified antibodies was evaluated by western blotting. The antibodies bound specifically to their target proteins producing 27 and $17 \mathrm{kDa}$ bands for $\alpha-\mathrm{OmpW}$ and $\alpha$-CtxB, respectively. No band was appeared by purified $\operatorname{IgY}$ from control group chicken (Figure 1). The activity of purified antibodies was measured from indirect ELISA absorbance data. The results of the purified IgY antibodies obtained from immunized chickens showed specific reaction to recombinant proteins. No specific antibodies against OmpW and $\mathrm{CtxB}$ antigens were seen in the control group ( $P$ value $<0.0001)$. Indirect ELISA assays indicated specific binding of $\alpha$-OmpW and $\alpha$-CtxB antibodies to the corresponding targets as expected. The production rate of specific $\alpha$-OmpW and $\alpha-\mathrm{CtxB}$ during the egg collection time is shown in Figure 2. According to the results, the antibody levels started to increase significantly two weeks after the final immunization $(P$ value $<0.05)$. The antibody titer peaked in the third week and remained at a relatively constant level until the end of egg collection (eighth week).

3.5. Optimization of Two Sandwich ELISAs. The sandwich ELISAs were optimized using the checkerboard method. According to the OD450 and positive/negative values, the ideal concentration of capture antibodies for coating were set at 1.25 and $0.6 \mu \mathrm{g} / \mathrm{mL}$ for $\alpha$-OmpW and $\alpha$-CtxB ELISAs, respectively (Figures 3(a) and 3(c)). The optimal dilution of HRP- $\alpha$-OmpW and biotin-conjugated anti- $V$. cholerae toxin $B$ subunit antibodies was $1: 16,000$ and $1: 32,000$, respectively (Figures $3(\mathrm{~b})$ and $3(\mathrm{~d})$ ).

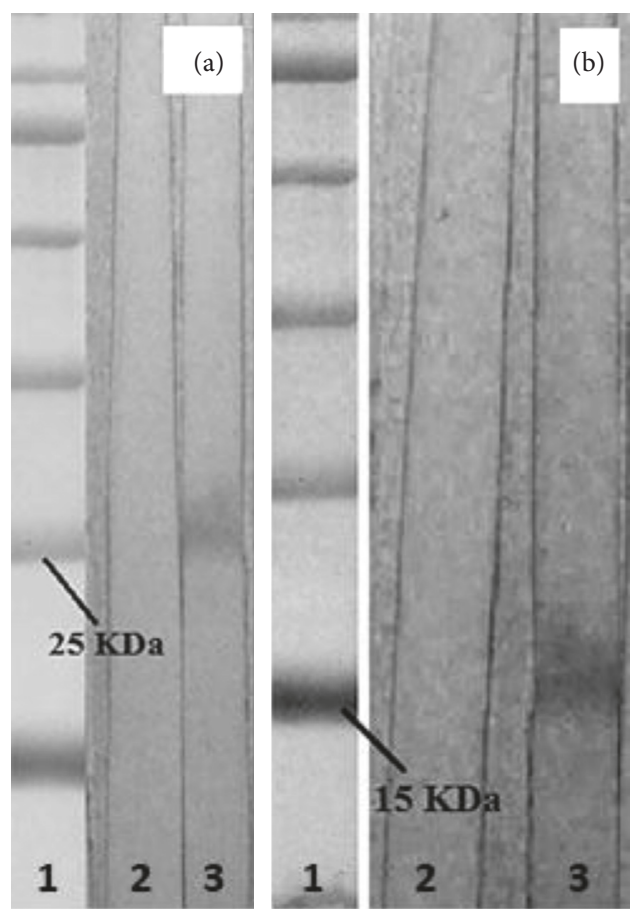

Figure 1: Binding capability evaluation of $\alpha$-OmpW and $\alpha$-CtxB antibodies. (a) Strip 1: protein size marker; Strip 2: purified IgY from control group chicken showed no band with recombinant OmpW; and Strip 3: the band of $27 \mathrm{kDa}$ recombinant OmpW reacted with $\alpha$-OmpW antibodies. (b) Strip 1: protein size marker; Strip 2: purified IgY from control group chicken showed no band with recombinant $\mathrm{CtxB}$; and Strip 3: the band of $17 \mathrm{kDa}$ recombinant $\mathrm{CtxB}$ reacted with $\alpha$-CtxB antibodies.

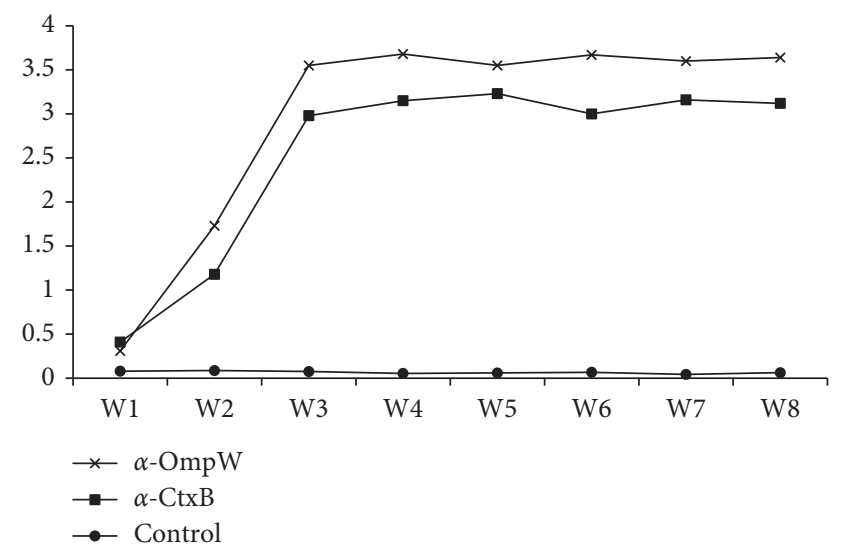

FIgURE 2: Changes in the specific IgY levels in the egg yolk after the last immunization in immunized and control groups.

3.6. Analytical Sensitivity and Specificity of the Developed Assays. Analytical sensitivity of the newly developed assays was determined by serial dilutions of $V$. cholerae and recombinant $\mathrm{CtxB}$ to construct a standard curve. The resulted equations were used to calculate the limit of detections. Table 3 shows the final results of the sensitivity evaluation. In addition, testing different bacterial strains (Table 1) with $\alpha$-OmpW and $\alpha$-CtxB ELISA did not show signals higher than their limit of blanks (OD: 0.254 and 


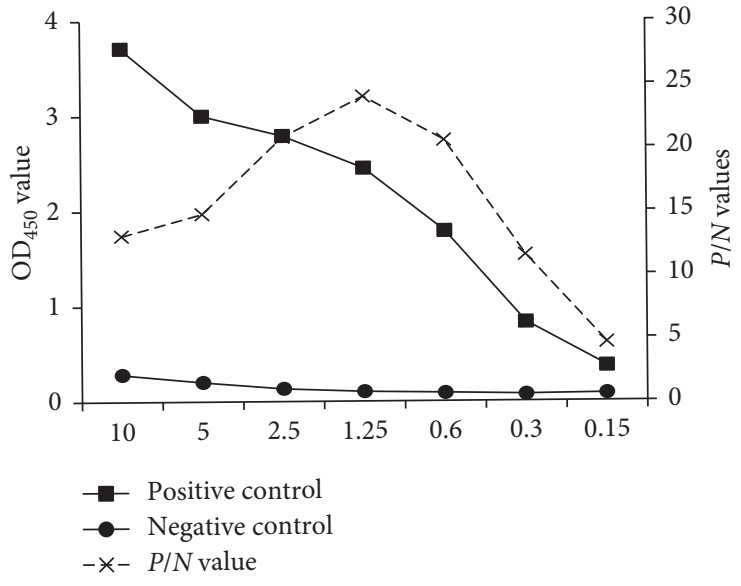

(a)

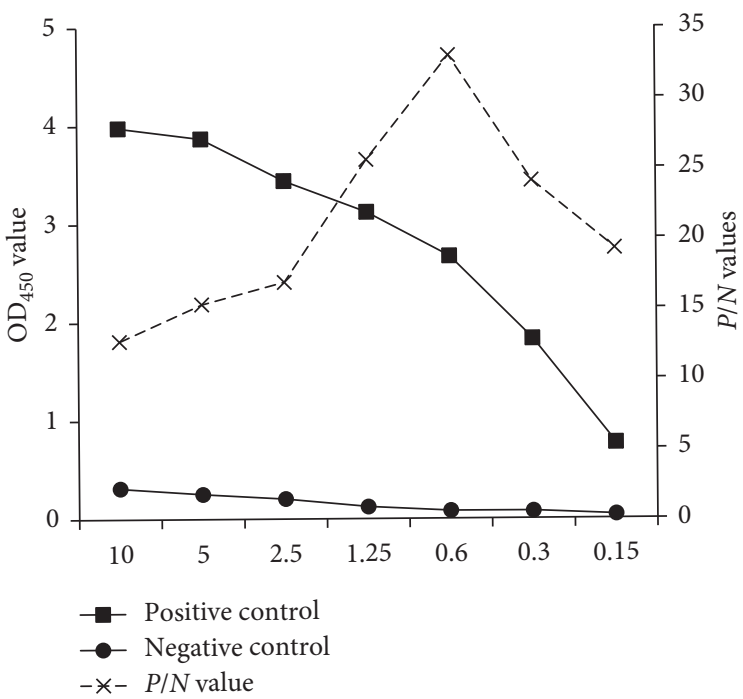

(c)

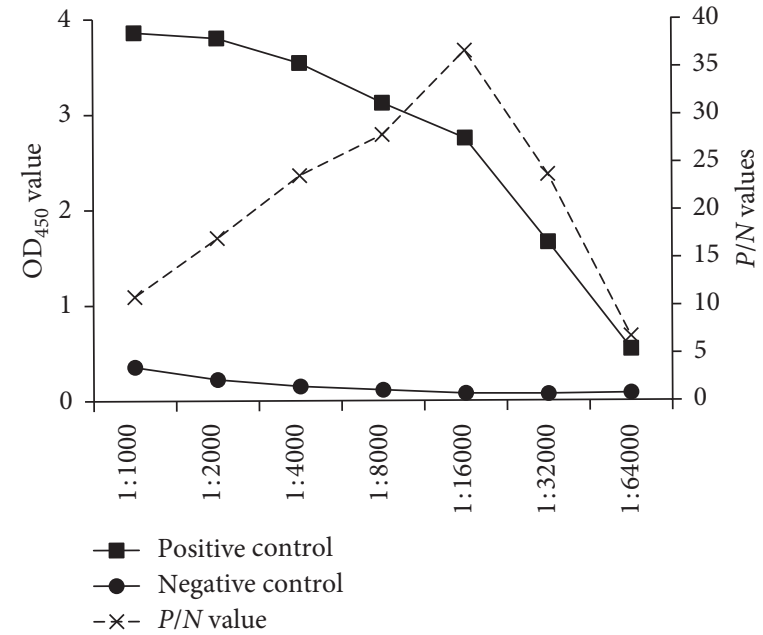

(b)

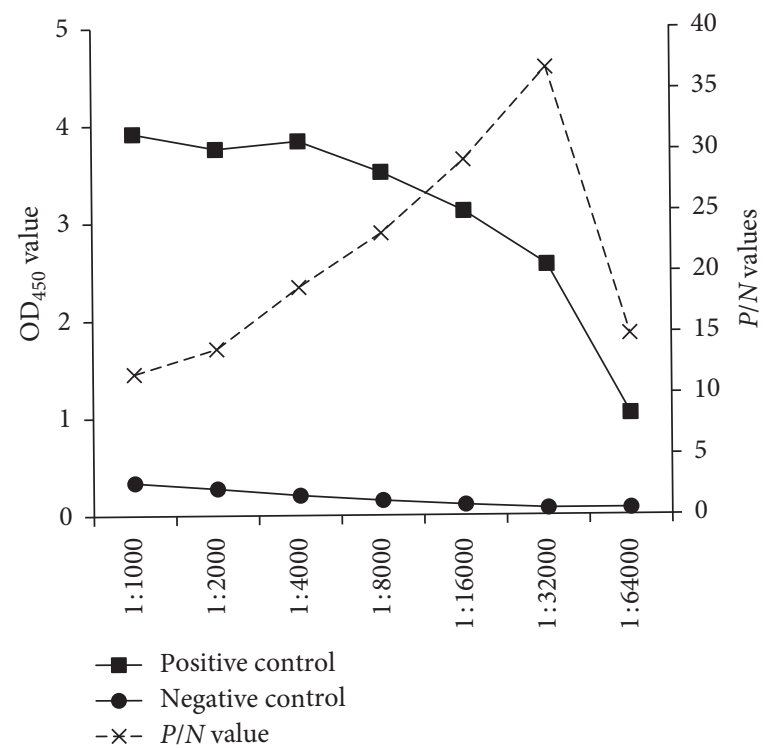

(d)

Figure 3: Optimization of $\alpha$-OmpW and $\alpha$-CtxB ELISAs. (a) Coating concentration of $\alpha$-OmpW and (b) HRP-labeled $\alpha$-OmpW dilutions. (c) Coating concentration of $\alpha-\mathrm{CtxB}$ and (d) biotin- $\alpha-\mathrm{CtxB}$ dilutions.

TABLE 3: The result of analytical sensitivity calculation for newly developed ELISAs.

\begin{tabular}{lcccccc}
\hline ELISA & Mean OD of blanks & SD of blanks & LOB & Absorbance of LoD & Line equation & LoD \\
\hline$\alpha$-OmpW & 0.110 & 0.087 & 0.254 & 0.373 & $y=0.1965 x-0.2103$ & $933 \mathrm{cfu} \cdot \mathrm{mL}^{-1}$ \\
$\alpha$-CtxB & 0.087 & 0.022 & 0.123 & 0.153 & $y=0.0031 x+0.0508$ & $33 \mathrm{pg} \cdot \mathrm{mL}^{-1}$ \\
\hline
\end{tabular}

OD: 0.123 , respectively), indicating no cross-reactivity with the tested bacteria. Testing the supernatant of $E$. coli (ETEC) culture grown under LT-toxin stimulation condition by $\alpha$-CtxB ELISA resulted in the mean optical density of 0.29 , which was higher than the absorbance of LoD of the assay.

3.7. Analytical Sensitivity and Specificity of the Assays in Spiked Stool Matrix Sample. In $\alpha$-OmpW, the spiked sample contained at least $7.5 \times 10^{3} \mathrm{cfu} / \mathrm{mL}$ of Vibrio chloerae showed an optical density (OD) greater than the limit of detection of the assay, i.e., 0.373. In CtxB ELISA, the optical density of the sample spiked with $62.5 \mathrm{pg} / \mathrm{mL}$ of recombinant CtxB was higher than the limit of detection of the assay, i.e., 0.153. In both assays, the final concentrations of the spiked samples are calculated by considering the dilution factor used for preparation of cleared sample. The results are shown in Table 4. 
TABLE 4: The result of analytical sensitivity and specificity of the developed ELISAs in spiked stool sample.

\begin{tabular}{|c|c|c|c|c|c|}
\hline \multicolumn{3}{|c|}{$\alpha$-OmpW } & \multicolumn{3}{|c|}{$\alpha-\mathrm{CtxB}$} \\
\hline Spiked conc. $\left(\mathrm{Cfu} \cdot \mathrm{mL}^{-1}\right)$ & Final conc. ${ }^{*}\left(\mathrm{cfu} \cdot \mathrm{mL}^{-1}\right)$ & OD & Spiked conc. $\left(\mathrm{pg} \cdot \mathrm{mL}^{-1}\right)$ & Final conc. ${ }^{*}\left(\mathrm{pg} \cdot \mathrm{mL}^{-1}\right)$ & OD \\
\hline $7.5 \times 10^{7}$ & $3.8 \times 10^{7}$ & 2.86 & 500 & 250 & 1.73 \\
\hline $7.5 \times 10^{6}$ & $3.8 \times 10^{6}$ & 1.92 & 250 & 125 & 1.02 \\
\hline $7.5 \times 10^{5}$ & $3.8 \times 10^{5}$ & 1.25 & 125 & 62.5 & 0.45 \\
\hline $7.5 \times 10^{4}$ & $3.8 \times 10^{4}$ & 0.69 & 62.5 & 31.3 & 0.21 \\
\hline $7.5 \times 10^{3}$ & $3.8 \times 10^{3}$ & 0.41 & 31.3 & 15.6 & 0.14 \\
\hline $7.5 \times 10^{2}$ & $3.8 \times 10^{2}$ & 0.18 & 15.6 & 7.8 & 0.08 \\
\hline $7.5 \times 10^{1}$ & $3.8 \times 10^{1}$ & 0.07 & 7.8 & 3.9 & 0.06 \\
\hline 0 (negative control) & 0 & 0.08 & 0 (negative control) & 0 & 0.06 \\
\hline
\end{tabular}

*Final concentration is calculated by considering the dilution factor used for preparation of cleared sample.

\section{Discussion}

Because of its rapid spread and vast epidemics, cholera associates with a heavy economic burden in the involved endemic area. According to the Global Health Observatory (GHO) data, in 2016, 132,121 cholera cases and 2420 deaths were reported to WHO worldwide [18]. Dealing with this threat necessitates paying more attention through improving global health. Vaccination and employing rapid diagnostic methods are two major solutions to achieve this goal. Early diagnosis of cholera outbreak leads to the prevention, control, and management of the disease. As a result, employing rapid, simple, and inexpensive diagnostic methods is considered as a very critical tool to control the spread of cholera [19].

Considering the need for a rapid diagnostic method, in this study, the avian antibodies targeting $V$. cholerae antigens have been produced to be used for the development of two sandwich ELISA methods, $\alpha$-OmpW and $\alpha$-CtxB ELISA, to be potentially used for the specific and sensitive detection of $V$. cholerae and its cytotoxin.

For this purpose, a specific polyclonal antibodiestargeting outer membrane protein $\mathrm{W}(\mathrm{OmpW})$ has been employed for the detection of $V$. cholerae. A comprehensive study on ompW gene conducted by Nandi et al. demonstrated that its coding sequence is highly conserved between $V$. cholerae strains and can be used for diagnostic purposes [10]. In addition, immunogenicity evaluation shows that OmpW is a strong immunogenic protein [20]. These two features make this antigen an ideal target to be used in diagnostic immunoassays. In addition, for discriminating toxigenic and nontoxigenic strains, subunit B of cholera toxin $(\mathrm{CtxB})$ was used. Cholera toxin is produced by the CTXф residing within the bacterial chromosome. The sequence of cytotoxin gene of CTXф is conserved in classic and El Tor strains, including some single-nucleotide polymorphisms [21]. In addition, there are some studies proving the suitability of CtxB as a specific marker for discrimination of toxigenic strains $[4,22]$.

Here, two avian polyclonal antibodies were raised against recombinant $\mathrm{OmpW}$ and $\mathrm{CtxB}$ antigens of $V$. cholerae. To the best of our knowledge, current study is the first report on using IgY in the detection of toxigenic $V$. cholerae. Avian egg antibodies are a very rich and costeffective source of these valuable molecules. Noninvasive sampling, high antibody amount (50-100 mg/egg), no interference with mammalian immunoglobulins (rheumatoid factors), HAMA (human anti-mouse antibody), and complement are the most important functional characteristics of avian antibodies [23]. Dose and molecular weight of antigen, the type of adjuvant, the route of administration, and the chicken age, breed, and keeping conditions all influence the immune response of the immunized chicken [24]. In the current study, we used the intramuscular injection of antigen formulated with Freund's adjuvant. Production of the specific antibodies started to increase two weeks after immunization $(P$ value $<0.05)$, peaked at the third week, and remained in constant level until the end of study (eighth week).

In this study, the egg yolk antibodies were extracted and purified by polyethylene glycol method [12]. There are different researches on IgY purification, reporting different yields of purified IgY. Pauly et al. reported the purification yields ranging from 28.8 to $60 \mathrm{mg}$ per egg yolk for different specific antibodies $[12,25]$. In another study for the production of IgY against soluble antigens of Toxoplasma gondii, the researchers were able to purify $48 \mathrm{mg}$ of antibody per egg [26]. According to their report, Wen et al. yielded $76.5 \mathrm{mg}$ of IgY antibody against inactivated influenza B virus [27]. Here, the average yield of purified $\alpha$-OmpW and $\alpha$-CtxB is 66.5 and $50.9 \mathrm{mg}$ per egg, respectively. The yield obtained in the present study is acceptable and comparable with the other similar experiments.

The purified specific IgY antibodies were used for the development of the two sandwich ELISAs. Performing checkerboard titration allowed a simple and quick optimization of the involved factors. Using purified IgY and applying precise optimization procedure led to development of the specific ELISAs with low detection limits, which have a significant advantage over the existing methods. Currently, various methods for the detection of $V$. cholerae have been introduced. In 2003, Pasteur Institute of Paris developed an immunochromatographic method based on antilipopolysaccharide antibodies. The detection limit of the assay was $10^{7} \mathrm{cfu} \cdot \mathrm{mL}^{-1}$ of $\mathrm{O} 1$ strain and $10^{6} \mathrm{cfu} \cdot \mathrm{mL}^{-1}$ of O139 [9]. In another research, Tuteja et al. designed a dipstick sandwich ELISA to detect OmpW and CtxB antigens. The analytical sensitivity of the developed assay was $10^{4} \mathrm{cfu} \cdot \mathrm{mL}^{-1}$ for OmpW and $60 \mathrm{pg} / \mathrm{mL}$ of CtxB [22]. Also in the immunochromatographic test strip designed by 
Yamasaki et al., the reported limit of detection was $10 \mathrm{ng} / \mathrm{mL}$ of cholera toxin [28]. In the current research, the limit of detection of $\mathrm{OmpW}$ is less than $1000 \mathrm{cfu} / \mathrm{mL}$ and that of $\mathrm{CtxB}$ is around $33 \mathrm{pg} / \mathrm{mL}$. Therefore, our newly developed assays show higher sensitivity than other similar works. The yolks of immunized chickens are a very rich source of IgY, which leads to obtaining high-purity antibodies and consequent high sensitivity of the developed immunoassays. Evaluation of analytical specificity by different bacterial strains (Table 1 ) with $\alpha$-OmpW and $\alpha$-CtxB ELISA did not show any cross-reactivity, but as the heat-labile toxin (LT) of enterotoxigenic E. coli (ETEC) shares around 80 percent homology with $V$. cholerae CT [29], we further evaluated nonspecific reaction of $\alpha$-CtxB ELISA in the supernatant of enterotoxigenic $E$. coli cultured under LT-toxin stimulation condition. The result of $\alpha$-CtxB ELISA in this condition shows cross-reactivity with $V$. cholerae cytotoxin. Increasing the LoD of $\alpha$-CtxB ELISA from 0.153 to a value above 0.29 would be helpful to overcome the problem resulting from the nonspecific reaction with ETEC enterotoxin. Considering the value of 0.30 for the LoD of $\alpha$-CtxB ELISA results in the limit of detection to be around $80 \mathrm{pg} / \mathrm{mL}$, which still shows an acceptable sensitivity for this test. On the other hand, as these ELISAs are considered to be used as two subsequent tests, only the specimens which contain $V$. cholerae (OmpW-positive samples) will be tested by $\alpha$-CtxB ELISA. This decreases the probability of cross reaction by ETEC enterotoxin.

The evaluation of the analytical sensitivity of the assays using spiked stool samples shows that the stool matrix does not lead to false-positive results in negative control, and their sensitivity are at least $7500 \mathrm{cfu} / \mathrm{mL}$ and $62.5 \mathrm{pg} / \mathrm{mL}$ for $\alpha$-OmpW and $\alpha$-CtxB ELISAs, respectively. The sensitivity of the assays using fecal sample decreased in comparison with the analytical sensitivity obtained by antigenic and bacterial suspensions in PBS, i.e., $1000 \mathrm{cfu} / \mathrm{mL}$ and $33 \mathrm{pg} / \mathrm{mL}$ for OmpW and CtxB, respectively. The main factor causing the decrease in sensitivity is the need to dilute stool specimen for preparing clear samples, which reduces the sensitivity of the assays by at least two times. As usual, pathogen load for $V$. cholerae in rice-water stool are between $10^{10}$ and $10^{12}$ colony-forming units per liter [30], even with this decreased sensitivity, $\alpha$-OmpW ELISA is still efficient for the detection of $V$. cholerae in infected individuals. In addition, this method can be performed on fecal samples cultured in alkaline peptone water for 4 hours. Using 4 -h-enriched fecal samples will significantly increase the sensitivity [6] and, in addition, it will eliminate the need for making clear samples. Furthermore, to use this method for environmental samples, there is no need for making clear samples, and thus, the sensitivity of the method will not be reduced.

High analytical sensitivity and specificity of the developed assays, proved in this study, show the potential usefulness of them to be applied for diagnostic purposes. Further evaluation of the assays requires clinical sensitivity and specificity in a large-field trial using a large number of human stool and environmental samples. We plan to determine these parameters in $\alpha$-OmpW and $\alpha$-CtxB ELISAs in the future.

\section{Conclusions}

Due to the unique biological characteristics, IgY antibodies promise to resolve some of the current technical issues in immunological assays. Based on these features, we produced specific IgY antibodies for the development of two IgY-based sandwich ELISAs for the specific and sensitive detection and discrimination of toxigenic $V$. cholerae. As the specificity of the designed assays is $100 \%$ and their sensitivities are higher than current rapid tests, they can be used as a reliable and robust tool to be potentially used for the detection of toxigenic $V$. cholerae in clinical and environmental samples. To the best of our knowledge, this study is the first report on using IgY in detection of $V$. cholerae.

\section{Data Availability}

The data used to support the findings of this study are available from the corresponding author upon request.

\section{Conflicts of Interest}

The authors declare that they have no conflicts of interest related to the material in the manuscript.

\section{References}

[1] B. Pang, M. Yan, Z. Cui et al., "Genetic diversity of toxigenic and nontoxigenic Vibrio cholerae serogroups $\mathrm{O} 1$ and $\mathrm{O} 139$ revealed by array-based comparative genomic hybridization," Journal of Bacteriology, vol. 189, no. 13, pp. 4837-4849, 2007.

[2] S. M. Faruque, M. J. Albert, and J. J. Mekalanos, "Epidemiology, genetics, and ecology of toxigenic Vibrio cholerae," Microbiology and Molecular Biology Reviews, vol. 62, no. 4, pp. 1301-1314, 1998.

[3] J. F. Mehrabadi, P. Morsali, H. R. Nejad et al., "Detection of toxigenic Vibrio cholerae with new multiplex PCR," Journal of Infection and Public Health, vol. 5, no. 3, pp. 263-267, 2012.

[4] J. P. Yadava, M. Jain, and A. K. Goel, "Detection and confirmation of toxigenic Vibrio cholerae $\mathrm{O} 1$ in environmental and clinical samples by a direct cell multiplex PCR," Water SA, vol. 39, no. 5, pp. 611-614, 2013.

[5] J. Frean, O. Perovic, V. Fensham et al., "External quality assessment of national public health laboratories in Africa, 2002-2009," Bulletin of the World Health Organization, vol. 90, no. 3, pp. 191-199, 2012.

[6] N. A. Bhuiyan, F. Qadri, A. S. Faruque et al., "Use of dipsticks for rapid diagnosis of cholera caused by Vibrio cholerae $\mathrm{O} 1$ and O139 from rectal swabs," Journal of Clinical Microbiology, vol. 41, no. 8, pp. 3939-3941, 2003.

[7] F. Qadri, A. Chowdhury, J. Hossain et al., "Development and evaluation of rapid monoclonal antibody-based coagglutination test for direct detection of Vibrio cholerae O139 synonym Bengal in stool samples," Journal of Clinical Microbiology, vol. 32, no. 6, pp. 1589-1590, 1994.

[8] Y. Uesaka, Y. Otsuka, M. Kashida et al., "Detection of cholera toxin by a highly sensitive bead-enzyme linked immunosorbent assay," Microbiology and Immunology, vol. 36, no. 1, pp. 43-53, 1992.

[9] F. Nato, A. Boutonnier, M. Rajerison et al., "One-step immunochromatographic dipstick tests for rapid detection of Vibrio cholerae O1 and O139 in stool samples," Clinical and Vaccine Immunology, vol. 10, no. 3, pp. 476-478, 2003. 
[10] B. Nandi, R. K. Nandy, S. Mukhopadhyay et al., "Rapid method for species-specific identification ofVibrio cholerae using primers targeted to the gene of outer membrane protein OmpW," Journal of Clinical Microbiology, vol. 38, no. 11, pp. 4145-4151, 2000.

[11] M. M. Bradford, "A rapid and sensitive method for the quantitation of microgram quantities of protein utilizing the principle of protein-dye binding," Analytical Biochemistry, vol. 72, no. 1-2, pp. 248-254, 1976.

[12] D. Pauly, P. A. Chacana, E. G. Calzado et al., "IgY technology: extraction of chicken antibodies from egg yolk by polyethylene glycol (PEG) precipitation," Journal of Visualized Experiments, vol. 1, no. 51, article e30, 2011.

[13] G. B. Wisdom, "Horseradish peroxidase labeling of IgG antibody," in The Protein Protocols Handbook, J. M. Walkerpp. 680-683, Humana Press, New York, NY, USA, 3rd edition, 2009.

[14] M. A. Iwanaga and T. H. Kuyyakanond, "Large production of cholera toxin by Vibrio cholerae $\mathrm{O} 1$ in yeast extract peptone water," Journal of Clinical Microbiology, vol. 25, no. 12, pp. 2314-2316, 1987.

[15] D. A. Armbruster and T. Pry, "Limit of blank, limit of detection and limit of quantitation," Clinical Biochemist Reviews, vol. 29, no. S1, pp. S49-S52, 2008.

[16] L. Zhu, J. He, X. Cao, K. Huang, Y. Luo, and W. Xu, "Development of a double-antibody sandwich ELISA for rapid detection of Bacillus Cereus in food," Scientific Reports, vol. 6, no. 1, article 16092, 2016.

[17] L. B. Rocha, C. Y. Ozaki, D. S. Horton et al., "Different assay conditions for detecting the production and release of heatlabile and heat-stable toxins in enterotoxigenic Escherichia coli isolates," Toxins, vol. 5, no. 12, pp. 2384-2402, 2013.

[18] World Health Organization, "Global Health Observatory (GHO) data Cholera Statistics 2016," October 2017, http:// www.who.int/gho/epidemic_diseases/cholera/en/.

[19] B. Sharifi-Mood and M. Metanat, "Diagnosis, clinical management, prevention, and control of cholera; a review study," International Journal of Infection, vol. 1, no. 1, article e18303, 2014.

[20] M. Fasihi-Ramandi, H. Ghobadi-Ghadikolaee, S. AhmadiRenani, and K. Ahmadi, "Serum anti-Vibrio cholerae immunoglobulin isotype in $\mathrm{BALB} / \mathrm{c}$ mice immunized with ompW-loaded chitosan," International Journal of Enteric Pathogens, vol. 4, no. 2, article e33137, 2016.

[21] E. J. Kim, D. Lee, S. H. Moon, C. H. Lee, and D. W. Kim, "CTX prophages in Vibrio cholerae O1 strains," Journal of Microbiology and Biotechnology, vol. 24, no. 6, pp. 725-731, 2014.

[22] U. Tuteja, S. Kumar, J. Shukla et al., "Simultaneous direct detection of toxigenic and non-toxigenic Vibrio cholerae from rectal swabs and environmental samples by sandwich ELISA," Journal of Medical Microbiology, vol. 56, no. 10, pp. 13401345, 2007.

[23] L. S. Munhoz, G. D. Vargas, G. Fischer et al., "Avian IgY antibodies: characteristics and applications in immunodiagnostic," Ciência Rural, vol. 44, no. 1, pp. 153-160, 2014.

[24] R. Schade, E. G. Calzado, R. Sarmiento et al., "Chicken egg yolk antibodies (IgY-technology): a review of progress in production and use in research and human and veterinary medicine," Alternatives to Laboratory Animals, vol. 33, no. 2, pp. 129-154, 2005.

[25] D. Pauly, M. Dorner, X. Zhang, A. Hlinak, B. Dorner, and R. Schade, "Monitoring of laying capacity, immunoglobulin Y concentration, and antibody titer development in chickens immunized with ricin and botulinum toxins over a two-year period," Poultry Science, vol. 88, no. 2, pp. 281-290, 2009.

[26] Á. F. Júnior, F. M. Santiago, M. V. Silva et al., "Production, characterization and applications for Toxoplasma gondiispecific polyclonal chicken egg yolk immunoglobulins," PLoS One, vol. 7, no. 7, Article ID e40391, 2012.

[27] J. Wen, S. Zhao, D. He, Y. Yang, Y. Li, and S. Zhu, "Preparation and characterization of egg yolk immunoglobulin $\mathrm{Y}$ specific to influenza B virus," Antiviral Research, vol. 93, no. 1, pp. 154-159, 2012.

[28] E. Yamasaki, R. Sakamoto, T. Matsumoto et al., "Development of an immunochromatographic test strip for detection of cholera toxin," BioMed Research International, vol. 2013, Article ID 679038, 7 pages, 2013.

[29] K. L. Roland, C. Cloninger, S. K. Kochi et al., "Construction and preclinical evaluation of recombinant Peru-15 expressing high levels of the cholera toxin B subunit as a vaccine against enterotoxigenic Escherichia coli," Vaccine, vol. 25, no. 51, pp. 8574-8584, 2007.

[30] E. J. Nelson, J. B. Harris, J. G. Morris Jr., S. B. Calderwood, and A. Camilli, "Cholera transmission: the host, pathogen and bacteriophage dynamic," Nature Reviews Microbiology, vol. 7, no. 10, pp. 693-702, 2009. 


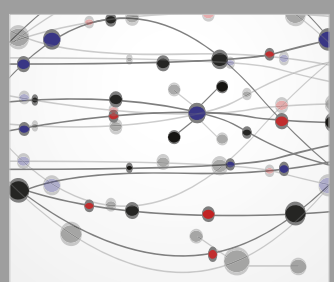

The Scientific World Journal
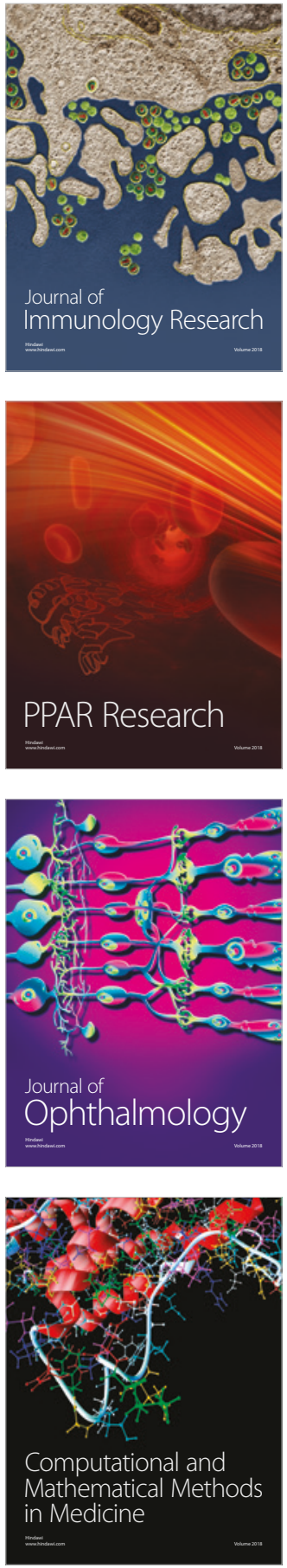

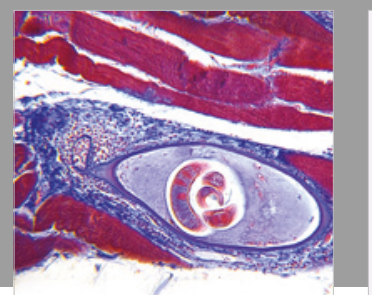

Gastroenterology Research and Practice

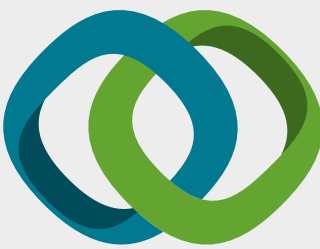

\section{Hindawi}

Submit your manuscripts at

www.hindawi.com
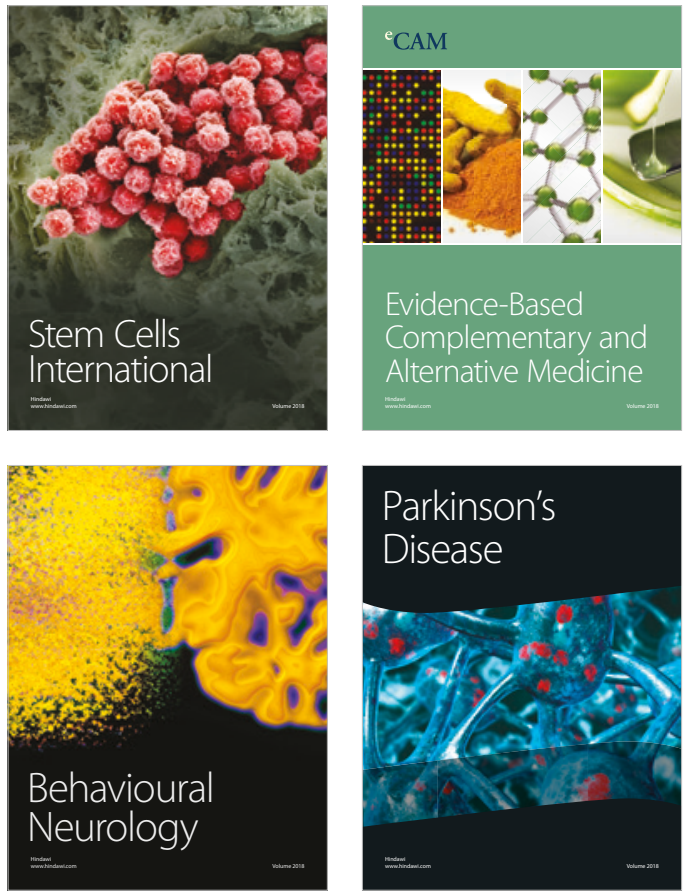

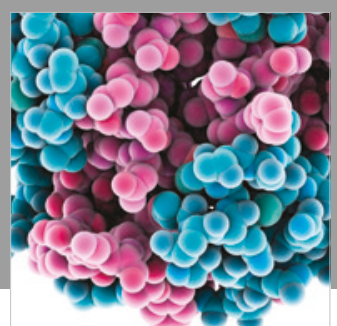

ournal of

Diabetes Research

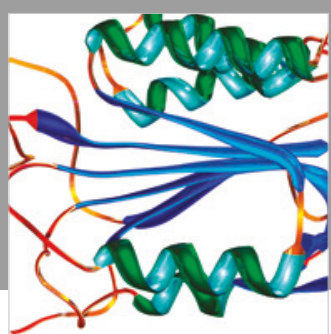

Disease Markers
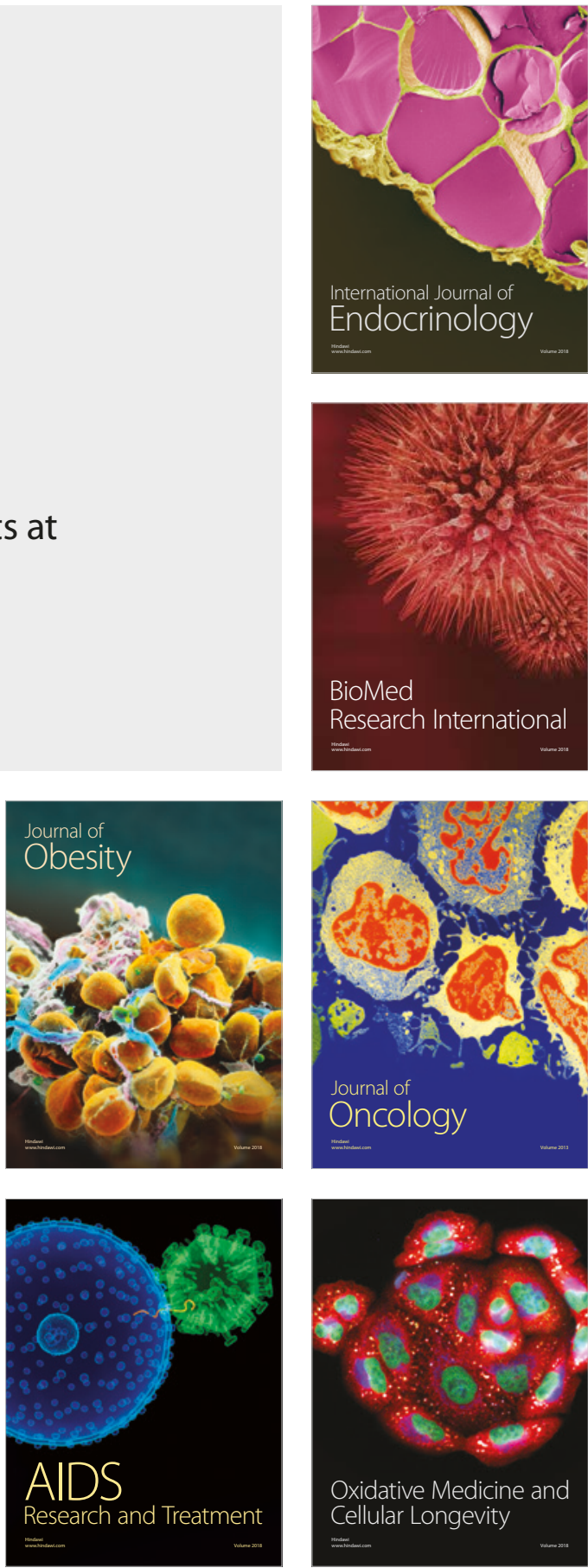